\title{
Subcutaneous transposition of the proximal oesophagus in oesophagoplasty
}

\author{
PA YANNOPOULOS
}

From Aretaion Hospital, Athens, Greece

ABSTRACT Experience with a new technique of oesophagoplasty is presented, in which the proximal part of the oesophagus is transposed to the subcutaneous space in front of the sternum and anastomosed to the organ replacing the oesophagus. This kind of operation has been performed successfully in five cases. The advantages are: (1) thoracotomy is avoided; (2) oesophageal anastomosis is easily performed; (3) anastomotic failure in the subcutaneous space is not a serious problem; (4) revision of the anastomosis is easily performed under local anaesthesia; and (5) the mortality rate due to oesophageal anastomotic failure is diminished. The procedure is indicated in cases where a long graft for oesophagoplasty is not available and intrathoracic anastomosis is not desirable. It may also be used after oesophagogastrectomy or total gastrectomy. The most common complication is the development of a salivary fistula that heals spontaneously in a few days.

Most surgeons believe that the oesophagus has a poor intramural blood supply and thus they avoid mobilisation during oesophageal surgery to prevent ischaemic necrosis and anastomotic failure. This concern may, however be exaggerated.

A continuous network of small arteries exists in the submucosa and over the external surface of the oesophagus, providing a good intramural blood supply. ${ }^{1}$ The efficacy of this network can be seen during oesophagectomy without thoracotomy. During this procedure, if the oesophagus is divided below the diaphragm and exteriorised through the cervical wound in continuity with the pharynx, although deprived of all its aortic oesophageal branches, its cut end bleeds, its colour remains normal, peristalsis is present, and bleeding from the mediastinum is minimal.

These observations indicate that the aortic oesophageal arteries may not be essential to the blood supply of this organ. If this is true, then subcutaneous transposition of the oesophagus should be a safe procedure, and in this article we report five cases in which this technique was used successfully.

Address for reprint requests: Dr P Yannopoulos, 2nd Surgical Department, Aretaion Hospital, University of Athens, 10 Ravine Street, Athens, Greece.

Accepted 14 June 1985

\section{Case reports}

CASE 1

A 63 year old man had an attack of acute pancreatitis. Two months later he developed a pancreatic pseudocyst and cystogastrostomy was performed through an upper midline incision. The postoperative course was complicated by gastric stasis and a nasogastric tube was left in place for many days. Because of the persistence of stasis an exploratory laparotomy was performed one month later and a fibrous stricture of the body of the stomach in the area of a previous gastrotomy was excised. After this operation the nasogastric tube was removed and the patient left hospital after a few days. When he started having an ordinary diet he experienced dysphagia that progressively worsened. The patient was admitted to the same hospital and a feeding jejunostomy was performed.

One month later he was referred to our unit, unable to swallow his own saliva. The oesophagogram showed a long stricture of the lower third of the oesophagus, which was resistant to dilatations. Oesophagoscopy showed a reflux stricture caused by the longstanding nasogastric tube. The patient also had poor respiratory function owing to emphysema and aspiration of saliva. We decided to perform an oesophagoplasty without entering the thorax, to reduce the postoperative complications that might be fatal for this high risk patient. 
At laparotomy the stomach was found to be unsuitable for oesophagoplasty because of the previous operations. The transverse colon and both colonic flexures, with the mesocolon, were contracted and adhering to the adjacent organs because of previous acute pancreatitis. The mesentery of the small intestine was also contracted, for the same reason. The only organ available for oesophagoplasty was the descending and sigmoid colon. We prepared a graft of this part of the colon nourished by the inferior mesenteric vessels but the graft was found to be too short to reach the neck and to be anastomosed to the cervical oesophagus.

To avoid thoracotomy we decided to transfer part of the oesophagus presternally to be anastomosed with the colon in the subcutaneous space. For this reason we divided it just above the cardioesophageal junction and closed its distal end in two layers. The thoracic oesophagus was dissected free from the posterior mediastinum without thoracotomy ${ }^{2}$ and exteriorised through a left cervical incision. The strictured part of the oesophagus was then excised. The exteriorised oesophagus, although completely deprived of its thoracic vessels, showed normal colour and peristalsis and its cut end was bleeding.

A subcutaneous tunnel was then created and an end to end oesophagocolonic anastomosis performed without any tension $8 \mathrm{~cm}$ below the sternal notch in the subcutaneous space (fig 1). The distal end of the graft was anastomosed to the stomach, end to side, and a colocolonic anastomosis performed.

The patient had a smooth postoperative course except for the development of a small salivary fistula from the oesophagocolonic anastomosis, which closed spontaneously in a few days. Eight months later he is able to take a regular diet.

\section{CASE 2}

A 65 year old woman was admitted to our hospital with an adenocarcinoma of the fundus of the stomach invading the lower oesophagus and oesophagogastrectomy was performed through an upper midline incision. The thoracic oesophagus was exteriorised through a left cervical incision. Its distal end was excised and $7 \mathrm{~cm}$ of the proximal oesophagus were positioned in front of the sternum subcutaneously and anastomosed end to end to the remaining part of the stomach. The patient had a smooth postoperative course and left hospital in a few days. Five months later she is able to take a regular diet.

\section{CASE 3}

A 70 year old man was admitted to our hospital with an adenocarcinoma of the fundus of the stomach invading the lower oesophagus and oesophagogastrectomy was performed through a midline inci-

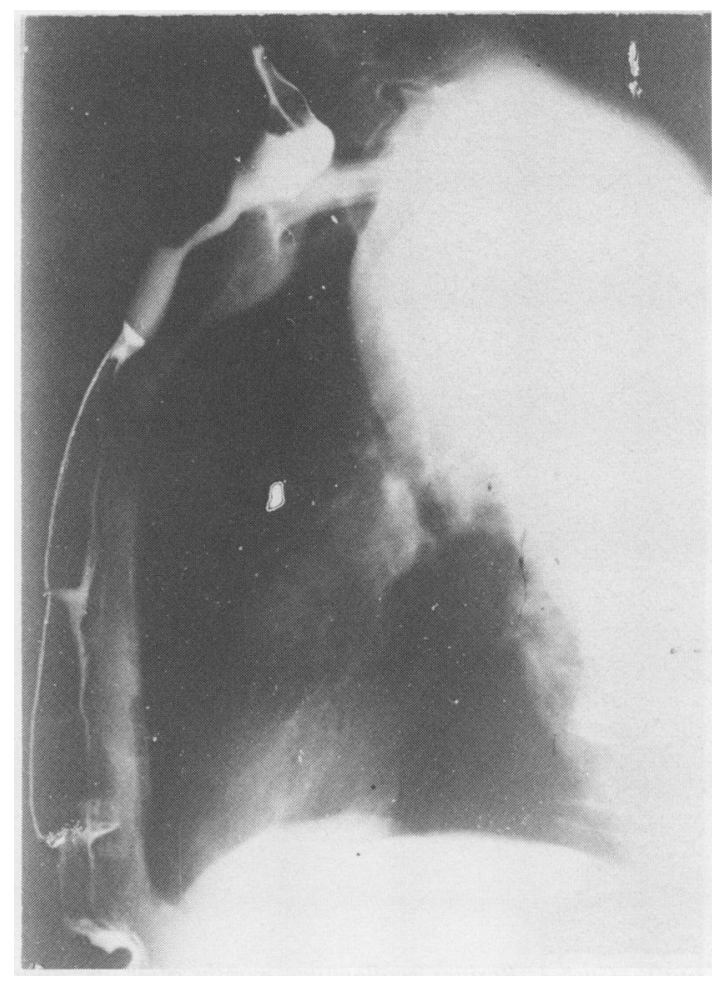

Fig 1 Case 1: Barium swallow six months after operation, showing the presternal oesophagocolonic anastomosis.

sion. The thoracic oesophagus was exteriorised through a left cervical incision. Its distal end was excised and $16 \mathrm{~cm}$ of the proximal oesophagus was positioned in front of the sternum subcutaneously and anastomosed end to end to the remaining part of stomach. The patient's postoperative course was smooth without any serious problems. He developed a small salivary fistula at the site of anastomosis that closed by itself in four weeks. Four months later he is able to take a regular diet (fig 2).

\section{CASE 4}

A 41 year old woman was admitted to our hospital because of a poorly differentiated adenocarcinoma of the stomach occupying most of this organ. A total gastrectomy was performed through an upper midline incision. The thoracic oesophagus was exteriorised through a left cervical incision. Its distal part was excised and $7 \mathrm{~cm}$ of the proximal oesophagus were placed subcutaneously in front of the sternum and anastomosed end to side to a Roux en Y jejunal loop. Her postoperative course was uneventful and she left the hospital in a few days. Two months later she is able to take a regular diet (fig 3 ). 


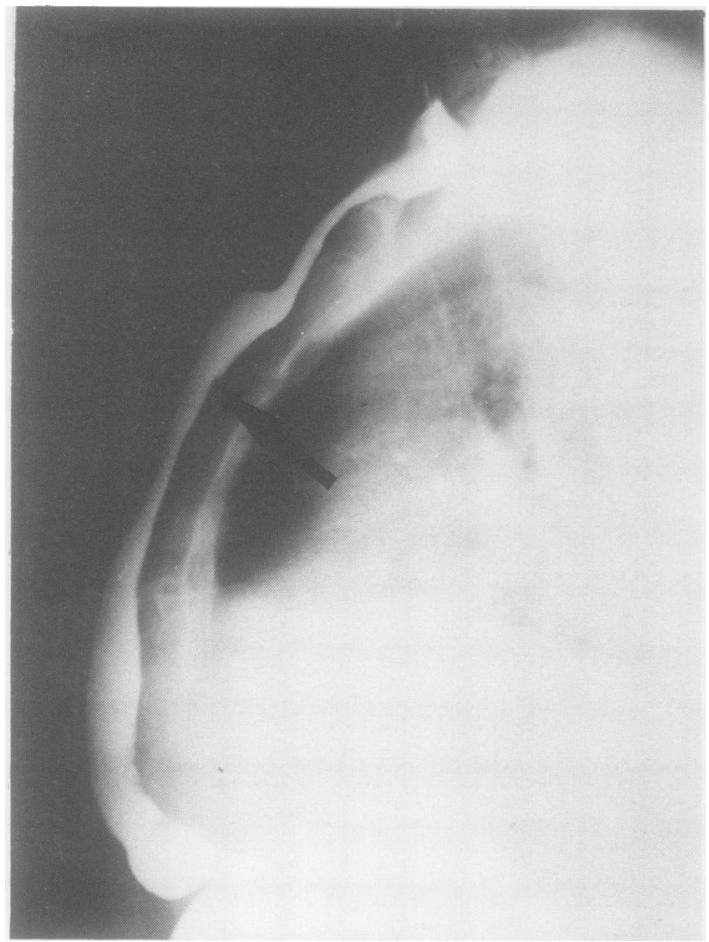

Fig 2 Case 3: Barium swallow two months after operation, showing the oesophagogastric anastomosis (arrow).

\section{CASE 5}

A 45 year old man was admitted to our hospital with severe dysphagia. Four months earlier he had had an oesophagogastrectomy with oesophagogastrostomy for carcinoma of the fundus of the stomach at another hospital. Oesophagoscopy showed a local recurrence at the anastomotic site. Computed tomography of the abdomen and thorax showed no metastases. The distal part of the oesophagus and the gastric remnant were excised through an upper midline incision. The thoracic oesophagus was exteriorised through a left cervical incision. Its distal part was excised and $8 \mathrm{~cm}$ of the proximal oesophagus were positioned subcutaneously in front of the sternum and anastomosed end to side to a Roux en $Y$ jejunal loop. The patient developed a small salivary fistula, which closed spontaneously in a few days. He left hospital able to take a regular diet.

\section{Discussion}

Subcutaneous transposition of the proximal part of the oesophagus seems to be a safe procedure. The oesophagus is nourished through a rich submucosal vascular network even after mobilisation and exteriorisation, when it remains in continuity with the pharynx.

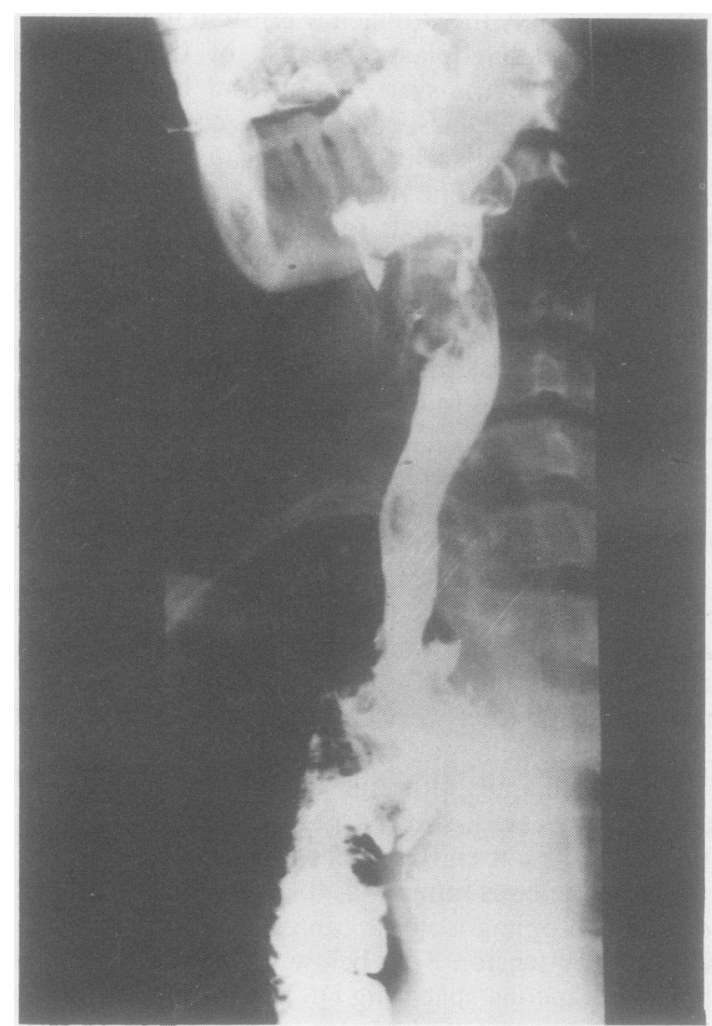

Fig 3 Case 4: Barium swallow one month after operation showing the oesophagojejunal anastomosis.

We have performed this kind of operation in five cases. The length of the exteriorised oesophagus (deprived of its thoracic vessels) ranged from 7 to $16 \mathrm{~cm}$. In all cases the transposed oesophagus showed no signs of ischaemia or necrosis. Three out of the five patients developed a salivary fistula at the anastomotic site that healed spontaneously and caused no serious problem to the patient. We cannot explain yet the high rate of this complication, but we do not think that is due to ischaemia, because its occurrence is not related to the length of the transposed oesophagus. The advantages of this procedure are obvious: (1) thoracotomy is avoided; (2) the oesophageal anastomosis is performed easily in the presternal region; (3) anastomotic failure, if it occurs in the subcutaneous space, does not create a serious problem; (4) revision of the anastomosis, if necessary, is easily performed under local anaesthesia; (5) the mortality rate due to oesophageal anastomotic failure, which is the main cause of death in patients with intra-abdominal or intrathoracic anastomoses, is reduced.

The operation is indicated in cases where a long graft for oesophagoplasty is not available and intrathoracic anastomosis is not desirable. It may also be 
used for benign or malignant lesions of the lower oesophagus; for malignant lesions of the cardia; and in total gastrectomy for carcinoma of the stomach, where a Roux en Y jejunal loop can be anastomosed to the oesophagus subcutaneously in the presternal region. The exact length of the oesophagus that can be deprived of its vessels without any sign of ischaemia remains to be determined. The criteria used in our cases were the normal colour of the transposed oesophagus, the presence of peristalsis, and bleeding of its cut end.

\section{References}

1 Ong GB. Resection and reconstruction of the esophagus. Curr Prob Surg 1971;3-56.

2 Orringer MC, Sloam H. Esophagectomy without thoracotomy. J Thorac Cardiovasc Surg 1978;76:643-54. 